\title{
Análisis de las experiencias de la Red de Microcines de Chaski (Perú) y Wordbytes de Worldwrite de Londres, en el desarrollo del sector audiovisual
}

Katherine Díaz Cervantes*

DOI: https://doi.org/10.33571/revistaluciernaga.v12n23a4

\section{Resumen}

El objetivo del artículo es analizar el rol que cumplen las organizaciones audiovisuales "middle ground". Para conseguirlo, se analizaron los objetivos y estrategias planteadas en los programas formativos: taller de cine comunitario de la Red de Microcines de Chaski de Lima - Perú, y el programa televisivo Wordbytes de Worldwrite de Londres - Inglaterra. En las organizaciones analizadas se reflejan las ciudades creativas en las que están ubicadas; coinciden en la búsqueda del desarrollo de públicos más críticos y creativos.

Palabras Clave: Industrias creativas; ciudades creativas; organizaciones fílmicas; audiovisuales; upperground; middleground; underground.

Recibido. Febrero 11,2020

Aceptado. Febrero 26, 2020

\footnotetext{
*Magíster Oficial de Gestión Cultural de la Universidad de Barcelona, con especialización en Patrimonio Mundial y Proyectos Culturales para el Desarrollo en la Universidad de Barcelona. Licenciada en Comunicación para el Desarrollo en la Pontificia Universidad Católica del Perú. Investigadora y gestora cultural en proyectos audiovisuales y gestión de archivos audiovisuales. Orcid: https://orcid.org/0000-0002-0600-9167 ; e-mail : katdcer@gmail.com
} 


\title{
Analysis of the experiences of the Chaski Microcines Network (Peru) and London Worldwrite Wordbytes, in the development of the audiovisual sector
}

\author{
Katherine Díaz Cervantes*
}

\section{DOI: https://doi.org/:10.33571/revistaluciernaga.v12n23a4}

\begin{abstract}
The principal objective of the article is analyses the role of the middle ground film organizations although the experiences of two particulars cases which are the Chaski Group (Lima) y Worldwrite (London). To achieve this, the objectives and strategies proposed by each organization are analyzed within their training programs for exploitation and exploration of content.

The organizations analyzed reflect the maturation of each of the creative cities where they live and coincide in the search for development of a new creative class, building more critical and creative audiences from the exploitation of new audiovisual content. Two organizations that have pursued their objectives through training programs such as the Community Film Workshop of the Microcines Network (Chaski) and the television program Wordbytes (Worldwrite).
\end{abstract}

Keywords Creatives Industries; creative cities; film or audiovisual organizations; upper ground; middle ground, underground.

Received. February 11, 2020

Accepted. February 26, 2020

*Master in Cultural Management in the University of Barcelona, with specialization in World Heritage and Cultural Projects for development in the University of Barcelona. Bachelor degree in Comunication for development in the Pontifical Catholic University of Peru. Researcher and cultural manager in audiovisual projects and audiovisual archives management. Orcid: https://orcid.org/0000-0002-0600-9167; email: katdcer@gmail.com 


\section{Análise das experiências da Rede dos Microcinemas Chaski do Peru e Worldwrite Wordbytes de Londes, no desenvolvimiento do sector audiovisual}

\section{Katherine Diaz Cervantes*}

DOI: https://doi.org/10.33571/revistaluciernaga.v12n23a4

\section{Resumo}

O objectivo do artigo é analisar o papel das organizações audiovisuais "intermediárias". Para o conseguir, foram analisados os objectivos e estratégias propostas nos programas de formação: seminários do cinema comunitário da Rede dos Microcinemas Chaski, em Lima - Peru, e o programa de televisão Wordbytes da Worldwrite em Londres - Inglaterra. As organizações analisadas reflectem as cidades criativas em que estão localizadas; coincidem na procura do desenvolvimento de públicos mais críticos e criativos.

Palavras-chave: Indústrias criativas; cidades criativas; organizações cinematográficas; audiovisuais; intermediárias.

Recebido. Fevereiro 11 de $2020 . \quad$ Aceite. Fevereiro 26 de 2020

*Mestre Oficial em Gestão Cultural na Universidade de Barcelona, com especialização em Património Mundial e Projectos Culturais para o Desenvolvimento na Universidade de Barcelona. Licenciatura em Comunicação para o Desenvolvimento na Universidades Pontificia Católica do Perú. Investigador e gestor cultural em projetos audiovisuais e gestão de arquivos audiovisuais. Orcid: https://orcid.org/0000-0002-0600-9167 ; e-mail: katdcer@gmail.com 


\section{Introducción}

Esta investigación partió del supuesto de que una organización sin fines de lucro middleground exitosa combina tanto las comunidades epistémicas como las comunidades de práctica. En el ámbito audiovisual, el papel que juega la organización middleground es de revitalizador o renovador de contenidos audiovisuales para generar mayor diversidad y pluralidad de visiones estéticas, artísticas y culturales. Se propuso como objetivo general analizar el rol que cumplen las organizaciones audiovisuales "middle ground" a través de las experiencias de dos casos particulares: grupo Chaski (Lima) y Worldwrite (Londres).
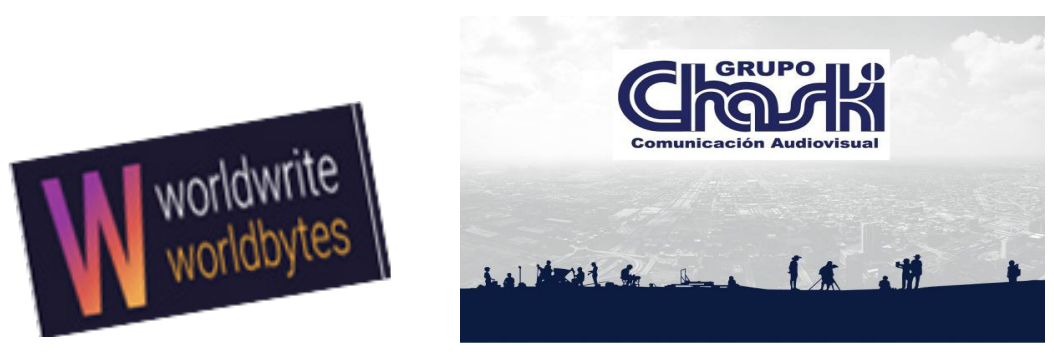

En específico se propuso analizar la teoría de la anatomía de las ciudades creativas, la cual clasifica las clases creativas como upperground, middleground, underground respectivamente. Colocando el énfasis en el componente middleground y sus dos tipos de comunidades: comunidades epistémicas y comunidades de práctica.

Un segundo objetivo específico que se contempló fue el de analizar las experiencias de dos organizaciones audiovisuales middleground (el grupo Chaski y Worldwrite) a través del analisis de sus objetivos y estrategias planteados dentro de sus programas formativos de explotación y exploración de contenidos para conocer mejor su impacto dentro del entorno.

La metodología para la investigación se basó en los marcos teóricos dados en la clase de "Ciudades Creativas" dictada por Monserrat Pareja Eastaway en el Máster de Gestión Cultural de la Universidad de Barcelona; fuentes bibliografías de revistas especializadas sobre economía y ciudades creativas (EIServier, Urban Studies y Sage Journals entre otras), en la cual han escrito especialistas en el campo de las ciudades creativas como Cohendet, Hall y Laundry.

La principal fuente de información ha sido la tesis de maestría "U+3 (i). Espacios para la emergencia y la fertilización de la creatividad: el caso de los festivales audiovisuales de Barcelona" (Vidaechea J., 2013) por aportar una gran variedad de marco teórico y analizar el comportamiento de las organizaciones en el ámbito audiovisual a través de tres componentes, representados por tres "i" que significan la oferta internacional, la interacción y el intercambio de conocimiento. 
Otra de las fuentes de información más consultadas han sido las páginas web oficiales de las dos organizaciones investigadas que son el Grupo Chaski (http://www.grupochaski.org/web/ ) y Worldwrite (http://www.worldwrite.org.uk/)

Una de las deficiencias de la investigación fue la dificultad para acceder a la información sobre el tema de ciudades creativas en el contexto de las organizaciones audiovisuales, en especial, dentro del concepto de "middleground".

El ámbito de estudio de esta investigación se limitó al sector audiovisual. En caso particular a las organizaciones audiovisuales sin fines de lucro cuyo objetivo es desarrollar una iniciativa social en la comunidad. Es importante resaltar que no tuvo la intención de comparar las dos industrias audiovisuales de estas ciudades creativas, ya que se consideró que las dos industrias audiovisuales (Lima y Londres) se encuentran en distintas etapas de desarrollo. Por una parte, Lima es una industria en pleno crecimiento; en cambio, Londres tiene un mercado consolidado siendo el Reino Unido uno de las cinco mayores industrias fílmicas de Europa (Talavera, 2017).

Del mismo modo, no es posible comparar las clases creativas de cada una de las ciudades. Pues se estableció que cada ciudad, tiene una clase creativa con mayor desarrollo en unos sectores que en otros. De manera general, los sectores culturales más desarrollados en el Perú son la danza, la artesanía y la gastronomía, logrando un gran reconocimiento internacional. En el caso particular del cine, es necesario reforzar la capacitación técnica y profesional y aumentar los recursos tecnológicos, en especial, en las zonas geográficas fuera de Lima. [1]

La industria cinematográfica peruana, localizada mayoritariamente en Lima, ha sido realmente favorable. En los últimos 6 años ha tenido crecimiento en cuanto a la producción, acercándose a un promedio de 40 estrenos anuales (en el 2013, eran 13 anuales) (Programa Ibermedia, 2020) [2].

En el 2017, el Congreso del Perú aprobó la nueva Ley de Cine (RPP, 2017), una reactualización de la Ley 26370, que ha dado como resultado que se brinden mayores recursos económicos. A fines del 2019, a través del Decreto de Urgencia № 022-2019, se otorgaron más de 25 millones de soles (7 millones de euros aproximadamente) (El Peruano, 2019). Los beneficios de esa nueva Ley se empezaron a notar en el año 2020 con un mayor estímulo económico para las producciones nacionales, una promoción a la educación cinematográfica y una mayor participación en las actividades de promoción y difusión audiovisual de parte del IRTP (Instituto Nacional de Radio y Televisión del Perú). (Caretas 23 de enero de 2020).

Se reitera que el objetivo de la investigación no fue comparar cual organización fílmica es mejor sino enfocarse en analizar cuáles son los objetivos y las estrategias que cada organización se plantea y si éstas son efectivas para lograr desarrollarse como una organización middleground. En otras palabras, cuáles han sido los elementos claves para el éxito o estancamiento de la organización como "middleground". 


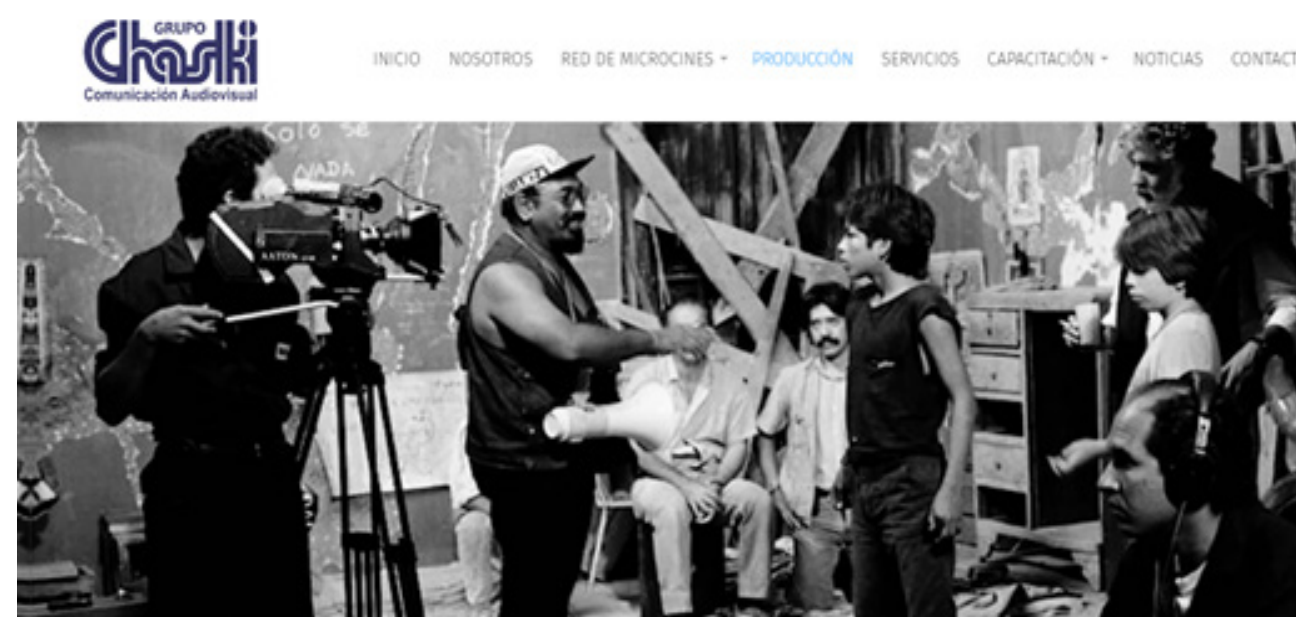

\section{Aproximaciones teóricas}

La definición de la ciudad creativa y de cuáles son sus características no tiene un consenso en la actualidad. Es un término relacionado con la economía e industria creativa y se describe como un modelo nuevo de ciudad que ha surgido debido a los efectos de la globalización y la innovación tecnológica.

Principalmente, las ciudades creativas se sustentan bajo la clase creativa, esto es una nueva clase de trabajadores altamente cualificados en tecnología y con talento innovador que son el motor para su desarrollo (Florida, 2010). Además, estas ciudades tienen un alto manejo de la transmisión de contenidos y una capacidad de almacenamiento en contenedores digitales o físicos (Hall, 2000). Por lo tanto, el componente de la creatividad es el método para explotar estos recursos y ayudarlos a crecer (Laundry, 2000).

En el campo cultural, estas ciudades altamente interconectadas desarrollan una red interdependiente entre los trabajadores, múltiples interacciones entre éstos y las compañías, una especialización en la producción y una aglomeración de recursos para su realización (Scott, 2006). Las ciudades creativas siempre han existido, desde la antigüedad, hasta ahora. Pasando por la antigua Roma, con la arquitectura clásica; Londres, con el teatro isabelino; o Los Ángeles con la industria del cine hollywoodense. Lo que ahora se presencia es un cambio en su estructura de organización y comunicación.

De manera general, la estructura de la ciudad creativa es vista como una serie de procesos de conocimientos entre individuos y compañías (o firmas). El análisis de Cohendet contribuye a identificar un tercer actor dentro de la estructura que son las comunidades (middle ground), que interconectan a los individuos con las firmas. "La formación de procesos creativos ha de ser entendida como la interacción entre los actores de las tres capas del territorio cuyo resultado es la creación de conocimiento" (Vidaechea J. , 2013, p. 40). Es interesante como Vidaechea prioriza los "procesos creativos" como determinante para la creación del conocimiento, que se antepone al "conocimiento creativo" de cada individuo.[4] 
En síntesis, la estructura de una ciudad creativa incluye estas tres niveles: upperground, middleground y underground. Estos son "permeables, complementarios, interdependientes y tienen una importancia relativa en el proceso de la producción del conocimiento conducente a la innovación que varía con el tiempo" (Vidaechea J. , 2013, p. 41).

Upperground, es el nivel mal alto de ciudad creativa. Está conformado por las empresas, instituciones, organizaciones, colectivos o comunidades del sector creativo; integrados por la "clase creativa", cuyo principal rol es brindan ideas al mercado. Su contribución al proceso creativo es "la capacidad de financiar y unir diferentes expresiones, integrar conocimiento disperso de diversas disciplinas y probar nuevas formas de creatividad en el mercado" (Cohendet et al, 2010, p. 95). Su dependencia con el entorno se debe a que carecen de departamentos de investigación o subsidiarias globales para acceder a ideas creativas, o redes globales para la participación de socios diversos.

En Barcelona existen varias organizaciones creativas, por ejemplo, "La Fábrica", con sedes en Barcelona y Madrid, reconocido por la realización del Festival Photoespaña y como proyecto innovador tiene el Festival de cine online Notodofilmfest.[5]

Middleground, es el nivel intermedio de la ciudad creativa, ubicado entre el upperground y underground. Este es fundamental en la estructura de una ciudad creativa y su posición privilegiada lo coloca "en entornos organizados formales e informales, promoviendo mecanismos de exploración como de explotación de la creatividad en procesos cognitivos que transcurren en ambas direcciones del eje vertical de la ciudad creativa (arriba-abajo) y (abajo-arriba)" (Vidaechea \& Pareja-Eastaway , 2017, p. 86).

La interconectividad característica del middleground genera dos tipos de comunidades que son identificadas como comunidades epistémicas y comunidades de práctica. La lógica de práctica de la comunidad epistémicas es de abajo a arriba; en el caso contrario, de las comunidades de práctica que es arriba a abajo.

La comunidad epistémica se enfoca en la explotación y codificación del nuevo conocimiento procedente del underground. Esta comunidad tiene como objetivo la creación de conocimiento que sea para el beneficio de la sociedad en general. Se diferencia a la comunidad en práctica por obedecer a una autoridad procesal común que es "un conjunto de códigos o reglas de conducta que define los objetivos de la comunidad, la guía de acción caracterizando los principios genéricos que cada miembro debe seguir" (Cohendet et al, 2009, p. 714).

La comunidad de práctica se enfoca en la explotación del conocimiento procedente del upperground. Esta comunidad realiza mecanismos de explotación de las ideas de las firmas u organizaciones como la sección de investigación aplicada del middleground. Su objetivo es codificar el conocimiento de las firmas para que sean más comprensibles al underground. Estas comunidades tienen una lógica de profesionalización de sus miembros para poder llevar a cabo el trabajo de constante codificación y explotación. 
El middleground conformado por las comunidades epistémicas y comunidades de practica requieren de "espacios, lugares y eventos específicos, que abran espacios de explotación y exploración, facilitando la experimentación y el intercambio de ideas y conceptos" (Vidaechea J. , 2013, p. 45).

Underground, es el nivel más bajo de la ciudad creativa. Está compuesto por individuos interesados en el arte y la cultura, cuyas actividades pueden estar desarrolladas por puro placer o para impulsar un sector artístico determinado. Es decir, que sus actividades no necesariamente pueden estar vinculadas con la producción, explotación o difusión del contenido cultural.

Este grupo es interesante pues "es una fuerza impulsadora de la definición de nuevas trayectorias evolucionarias de las artes y la cultura" y "es común que las empresas creativas se inspiren de las experimentaciones del underground" (Cohendet et al, 2010, p.96). Un ejemplo interesante es el caso del arte pictórico callejero de la cuidad de Los Ángeles que es relatado en el falso documental "Exit Through the Gift Shop". Este documental critica el mercado del arte y la innovación en el arte pop. También nos invita a conocer al artista "underground" Bansky, un personaje enigmático que siempre causa tendencia en los medios digitales y tradicionales, por las intervenciones urbanas que realiza por todo el mundo.[6].

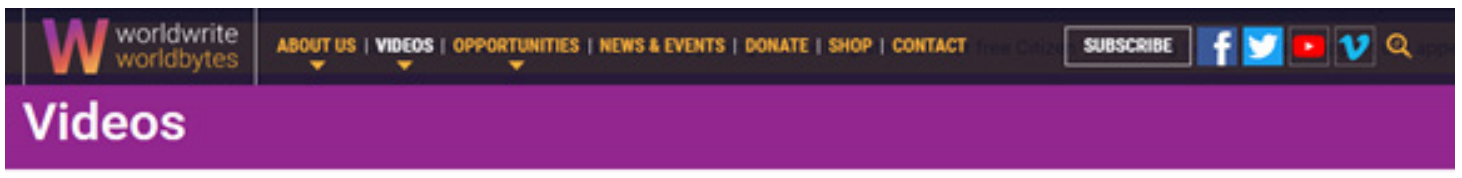

Civil Liberties ,
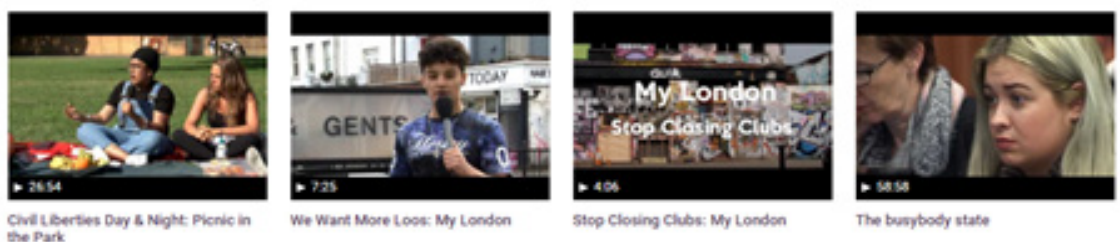

View more ,

Civibertion

We Want More Loos: My London

Stop clowing clubs: My Londer

The buspogofy stake

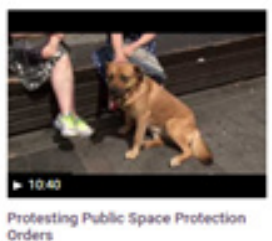

\section{Descripción de las organizaciones audiovisuales middleground}

\subsection{Grupo Chaski, Comunicación Audiovisual}

El Grupo Chaski es una organización sin fines de lucro creado en 1982, con sede principal en la ciudad de Lima, capital del Perú. Esta organización está compuesta por un grupo de reconocidos cineastas, comunicadores audiovisuales y gestores culturales "comprometidos con la promoción del cine como herramienta para el desarrollo cultural y educativo del país" (Grupo Chaski, 2020a). 


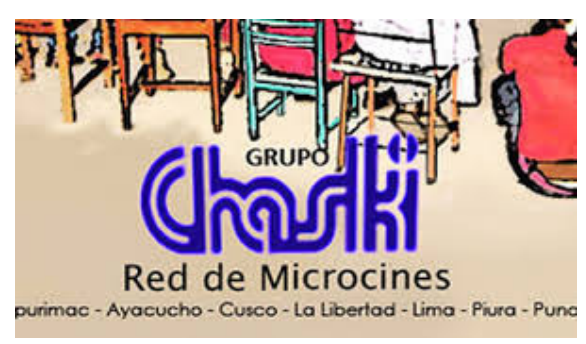

Esta organización promueve la democratización cultural a través del lenguaje audiovisual por medio de las redes de salas de cine alternativo llamados "microcines" que tiene once sedes alrededor del país.[7]. Este proyecto de redes sirve como espacio de cine comunitario y, a su vez, de un espacio de exhibición alternativo, en lugares del país donde no existen cines comerciales. Este es el principal proyecto que tiene el grupo Chaski y que se ha mantenido hasta la actualidad, siendo el único proyecto social que tenga una estructura de redes en el Perú.

La visión social del Grupo Chaski es apoyar en el crecimiento del conocimiento para el beneficio de la sociedad en general, orientándose en el fortalecimiento de las ideas, valores sociales y trasmisión de conocimientos o saberes culturales. Los contenidos que difunden en sus microcines buscan reflejar la realidad en la que viven y desarrollar un sentido crítico en su entorno y en la sociedad en general.

El público objetivo, en general, es todo el mundo. Y, de manera particular, son los jóvenes interesados en ver el cine y la experiencia de verlo fuera de casa y en comunidad. Además de desarrollar una mirada más crítica de su entorno próximo.

Desde el 2010, en el marco del proyecto de Red Nacional de Microcines, se comenzó el taller de cine comunitario Chaski para realizar producciones audiovisuales basadas en una metodología participativa que registraron "el corazón y pensamiento de los barrios, comunidades campesinas y organizaciones de las 11 regiones del Perú que componen la Red de Microcines" Este taller comunitario duró unos 5 años y recibieron premios internacionales en festivales internacionales (Grupo Chaski, 2015).

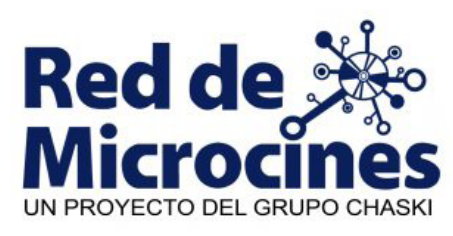

El grupo Chaski, como empresa productora audiovisual, tiene un importante reconocimiento a nivel nacional e internacional con los largometrajes de "Juliana" (1989) y "Gregorio" (1984). Los temas de sus películas abordaban problemas sociales. Como es el caso de Gregorio con el tema de la inmigración y el racismo; y, Juliana, con la marginalidad urbana y el rol de la mujer. Estas dos películas tuvieron una respuesta favorable en el público y números premios en festivales internacionales como La Habana, Biarritz, Huelva y Cartagena (Grupo Chaski, 2020b). 
Paralelamente, organizan el Encuentro Internacional de Cine Comunitario "Chacchando Sueños". Éste busca difundir y generar mayor crecimiento en la producción de cine comunitario, a través de nuevas tecnologías, de la gente que vive en los pueblos y barrios. Se concibe el cine comunitario aquel que en cada proceso de la realización del producto audiovisual refleje la participación activa vecinal, barrial o comunal. Para incentivar la vinculación de los jóvenes tiene un premio especial llamado "Jóvenes creativos" (Grupo Chaski, 2020c).

El financiamiento de sus largometrajes y proyectos artísticos y culturales ha sido por medio de subvenciones del Estado y auspiciadores. Grupo Chaski ha sido el ganador durante tres años (2017, 2018 y 2020) del Concurso de Gestión Cultural de los Estímulos Económicos para la actividad cinematográfica y audiovisual.

En el rubro de las firmas o socios privados está la fundación Liechtenstein Development Service (LED) y la federación internacional Terres des Hommes que colabora con los proyectos que organiza el Grupo Chaski.

\subsection{Worldwrite}

Fundada hace 26 años, Worldwrite es una organización sin fines de lucro, ubicada en la ciudad de Londres, capital de uno de los más importantes nodos culturales de la industria del cine europeo y mundial.

Esta organización tiene su sede en la ciudad de Londres y mantiene una participación constante con la comunidad de su alrededor, en especial, con los jóvenes. La visión de la organización es generar la democratización de la educación para el desarrollo igualitario de la sociedad, a través de la producción y difusión de contenidos audiovisuales de calidad (Worldwrite, 2020a).

Esta organización tiene como objetivo generar una conciencia crítica, a través del diálogo, con los jóvenes como protagonistas de las discusiones en temáticas generales que afectan la ciudad de Londres (inmigración, multiculturalidad, economía, historia, cambios sociales, progresos de la ciencia, etc..). Es un trabajo comunitario voluntario que los jóvenes realizan para la exploración y explotación de sus conocimientos. La labor de la organización también abarca la difusión de los contenidos a través de sus plataformas digitales por medio de su página web oficial.

El principal proyecto de Worldwrite es una Worldbytes denominado como un canal de televisión para la ciudadanía, de manera online y sin fines de lucro. Este proyecto produce contenidos televisivos online en donde los jóvenes no sólo participan en su realización sino que son proconsumers, es decir, protagonistas de los debates o discusiones en torno a los temas tratados. Este proyecto ganó el Premio "Niños y Jóvenes de ahora. Artes y Cultura" (Children and Young People Now Arts and Culture Award) en el 2018 (Worldwrite, 2020a). 
El público objetivo son jóvenes voluntarios, entre 16 a 25 años, que tengan tiempo libre los fines de semana. La organización no juzga ni la procedencia, ni el uso del idioma inglés o la experiencia previa en el campo audiovisual. Los jóvenes son capacitados en las herramientas para la creación y realización de contenidos audiovisuales online, participando tanto en la investigación, el manejo de cámara, sonido y técnicas de presentación y promoción (Worldwrite, 2020b). Luego de su capacitación, se les orienta y prepara para la realización de sus propios proyectos con una mirada crítica social.

Paralelamente, Worldwrite tiene colaboraciones con otras organizaciones para realizar proyectos de investigación y exploración de los contenidos como, por ejemplo, "La Batalla de las Ideas" (Battles of Ideas Festival, 2020), un evento que invita a investigadores, académicos, especialistas para discutir en una mesa de diálogo sobre distintos temas coyunturales.

Worldwrite son conocidos a nivel nacional por ser los pioneros en la televisión ciudadana en Reino Unido. Sus programas más conocidos son: 'Don't shout at the telly', 'Books and buns' $y$ 'Views on the streets' que reflejan una manera lúdica de acerca a la población desde un lenguaje coloquial. A la par, ha sido productora de diversos documentales galardonados, entre las que destacan, "Sylvia Pankhurst: Everything is Possible" (2011), "C.L.R. James" (2016), "1917: Why The Russian Revolution Matters" (2017) y "Women; a success story" (2018) (Worldwrite, 2020c).

El financiamiento de Wordwrite es amplio y variado abarcando los ámbitos públicos, privados y sin fines de lucro. La mayoría están ubicados o tienen sede en Londres. Se destaca las fundaciones (Esmée Fairbairn, Jack Petchey y Trust for London), la subvención nacional (Heritage Lottery Fund), las compañias privadas como Starbucks Youth Action, Bloomberg y Ford Britain Trust (Worldwrite, 2020d).

\section{Análisis de las experiencias de organizaciones audiovisuales middleground}

A continuación, describiremos los puntos fuertes y débiles de las organizaciones "middleground", tomando en cuenta los objetivos de cada una.

En el caso del Grupo Chaski, se identificaron procesos creativos que van de abajo hacia arriba (comunidades epistémicas). En donde se exploran los conocimientos de la comunidad local [8] para luego ser codificadas en un lenguaje audiovisual que es trasmitido a la comunidad audiovisual. El proyecto de los talleres de Cine Comunitario es un claro ejemplo de la construcción colectiva de conocimiento. En el caso del Encuentro Internacional de Cine Comunitario "Chacchando Sueños" es un espacio de encuentro entre los individuos y la comunidad para explorar y compartir ideas. 
El primer punto fuerte identificado es su constante exploración del contenido desde la búsqueda de un lenguaje audiovisual de calidad. Los objetivos de la organización justifican la codificación del conocimiento porque contribuyen a una mayor democratización de la cultural para el beneficio general de la sociedad. Éste se realiza por medio de la trasmisión de saberes, conservación de tradiciones culturales y promoción de la diversidad cultural.

El segundo punto fuerte es la Red de Microcines que representa un espacio de exhibición cinematográfica alternativa, en especial, en aquellos lugares donde no existe ningún espacio. Lo que lo posiciona en un lugar privilegiado en la exploración de conocimientos desde el underground. Luego de las secciones de cines, se generan debates o conversaciones en los que se recogen las impresiones del público y estos comparan los contenidos con su realidad cotidiana. Un tercer punto fuerte es el concepto de cine comunitario en el que la comunidad participa en la exploración y la codificación de los contenidos.

Como puntos débiles podemos identificar, en primer lugar, que el Grupo Chaski, en los procesos creativos de exploración, tiene capacidad limita para interpretar los conocimientos desde abajo hacia arriba. Siendo un trabajo arduo debido a que la sociedad necesita ser entendida desde sus propios idiomas o códigos que son tan diversos como las culturales del Perú.

En segundo lugar, los objetivos planteados buscan mejorar la visión crítica del público, pero no logran tener un mayor alcance. Es decir, preparan a los jóvenes para ser sólo consumidores y espectadores de cine.

Los objetivos son parcialmente efectivos, pues el público necesita también herramientas para la explotación de su conocimiento. No se conocieron resultados de los talleres comunitarios, de su impacto y las razones de su falta de continuidad. La organización ha priorizado el Encuentro Internacional de Cine Comunitario. Un tercer y último punto débil es la falta de focalización en un territorio determinado pues el proyecto de Microcines se situada alrededor 11 zonas de distintas del país.

En cuanto a WorldWrite consideramos que esta organización middleground, realiza procesos creativos que fluctúan entre las comunidades de prácticas y epistémicas. Explora y codifica el conocimiento de un territorio, al mismo tiempo, capacita o profesionaliza a los voluntarios para ser investigadores y creadores de sus propios contenidos audiovisuales, convirtiéndolos en personas cualificadas en el medio audiovisual (clase creativa).

El primer punto fuerte identificado es la inserción de tecnologías de la información como elemento innovador de esta organización. El proyecto Worldbytes se hace único en el mercado por el hecho de transmitir su contenido audiovisual de manera online. Otras organizaciones complementan sus contenidos como Battle of Ideas. 
En los procesos de codificación de contenidos han clasificado e identificado temas coyunturales que generan debate y reflexión sobre la situación actual de la ciudad (libertades civiles, democracia, economía, globalización, historia...).

Un segundo punto fuerte en la organización es su ubicación territorial, en tanto Londres cuenta con oferta cultural diversa que atrae a los jóvenes.

En tercer lugar se destaca la creación de prosumidores jóvenes que trabajan voluntariamente en el proceso de exploración y explotación del conocimiento de la ciudad. Lo que genera una doble vía de comunicación entre éstos y la ciudad. Ellos interactúan con la ciudad mientras investigan y luego procesan esta información para codificarla en términos de contenidos audiovisuales. Al mismo tiempo, los habitantes de la ciudad reflexionan y comunican sus vivencias a los voluntarios y espectadores en general.

Entre los puntos débiles de la organización está el carácter volátil de los voluntarios. Aunque se entiende la oportunidad de iniciar a los jóvenes como voluntarios, es de anotar que éstos requieren algún tipo de beneficio económico, premios o incentivos.

Otra de las debilidades es que el programa Worldbytes, sólo presenta contenidos en inglés, aunque en éste participan jóvenes anglohablantes y el canal pretende ser global.

Un tercer punto débil es la calidad de los contenidos. No queda claro si éstos son evaluados por investigadores o especialistas o que los jóvenes hayan sido orientados en la preparación de los temas.

\section{Conclusiones}

Las organizaciones middleground de Lima -Perú y Londres- Inglaterra, se han desarrollado con procesos creativos distintos y complementarios. El Grupo Chaski en Lima, está organizado mediante una amplia red de sedes que le otorga presencia territorial. Por su parte Worldbytes en Londres tiene un único nodo cultural con una alta capacidad de alcance a través de las tecnologías de la comunicación e información

En relación a los objetivos de cada organización, en el caso de Lima, si la organización está buscando espectadores más críticos y cultos cinematográficamente, necesita reforzar la educación cinematográfica, incentivando la creación de espacios de debates o reflexión. Estos deberían estar organizados conjuntamente con investigadores y especialistas, en el marco de proyectos complementarios que nutran las capacidades de los espectadores de los Microcines.

Los objetivos de la organización de Londres son claros y efectivos. En especial, en los procesos creativos donde los jóvenes realizan investigaciones de campo dentro de su ciudad para la elaboración de sus contenidos televisivos. En ese proceso se identificaron dos dimensiones, en la cual los jóvenes son los codificadores de los contenidos que transmiten los ciudadanos londinenses; 
y, del mismo modo, la organización codifica la información que los voluntarios les transmiten a través de un lenguaje audiovisual online.

Chaski y Worldwrite, reflejan el estado de maduración de cada una de las ciudades de donde se localizan. Siendo Lima y Londres dos ciudades atractivas culturalmente que seguirán desarrollándose de acuerdo de sus propias necesidades.

Estas dos organizaciones, a pesar de contar con procesos distintos, tienen como finalidad el desarrollo de una nueva clase de público más critico, creativo y empoderado que realice sus propios contenidos audiovisuales. En el caso de Lima, el apoyo del Estado y el aumento de las producciones cinematográficas ha generado un panorama optimista para el sector. En cuanto a Londres, su consolidación como ciudad creativa esta cimentada por un mercado fílmico maduro que se ha mantenido estable y duradero a lo largo de los años.

\section{Bibliografía}

Battles of Ideas Festival. (2020). Recuperado el 30 de Octubre de 2020, de https://www.battleofideas.org.uk/ Casani, F., Rodríguez-Pomeda, J., \& Sánchez, F. (2012). Los nuevos modelos de negocio en la economía creativa: Emociones y redes sociales. Universia Business Review(33), 48-99. Recuperado de https://www. redalyc.org/articulo. $.0 a ? \mathrm{id}=43323186003$

Cinencuentro. (8 de Enero de 2020). 51 películas peruanas se estrenaron el 2019. Recuperado el 24 de Octubre de 2020, de Cinencuentro Web Site: https:/www.cinencuentro.com/2020/01/08/50-peliculasperuanas-se-estrenaron-el-2019/

Cohendet, P., Grandadam, D., \& Simon, L. (2009). Economics and the ecology of creativity: evidence from the popular music industry. International Review of Applied Economics, 709-722. doi:https://doi. org/10.1080/02692170903239879

Cohendet, P., Grandadam, P., \& Simon, L. (2010). The Anatomy of the Creative City. Industry and Innovation, 91-111. doi:https://doi.org/10.1080/13662710903573869

Cohendet, P., Grandadam, P., \& Simon, L. (2011). Rethinking urban creativity: Lessons from Barcelona and Montreal. EIServier, 151-158. doi:https://doi.org/10.1016/j.ccs.2011.06.001

DAFO: Dirección del Audiovisual, la Fonografía y los Nuevos Medios. (6 de diciembre de 2017). Recuperado el 24 de Octubre de 2020, de http://dafo.cultura.pe/ministerio-de-cultura-reconocera-a-87-empresascinematograficas-de-todo-el-peru-en-ceremonia-de-premiacion/

El Peruano. (9 de Diciembre de 2019). Nueva ley impulsará cine peruano. Recuperado el 29 de Octubre de 2020, de https://elperuano.pe/noticia/87379-nueva-ley-impulsara-cine-peruano

Florida, R. (2010). La clase creativa. La transformación de la cultura del trabajo y el ocio en el siglo XXI. Barcelona: Paidós. 
Grupo Chaski. Comunicación Audiovisual. (2020a). Nosotros. Recuperado el 24 de Octubre de 2020, de Grupo Chaski Comunicación Audiovisual Web site: http://www.grupochaski.org/web/nosotros/

Grupo Chaski. Comunicación Audiovisual. (2020b). Producción Cinematográfica. Recuperado el 24 de Octubre de 2020, de Grupo Chaski. Comunicación Audiovisual Web site: http://www.grupochaski.org/web/ produccion-cinematografica/

Grupo Chaski. Comunicación Audiovisual. (2020c). Convocatoria «Chancchando Sueños». Recuperado el 2020 de Octubre de 30 , de http://www.grupochaski.org/web/convocatoria-chacchando-suenos/

Hall, P. (2000). Creative Cities and Economic Development. Urban Studies, 37(4), 639-649. doi:https://doi.org/10.1080/00420980050003946

Laundry, C. (2000). The Creative City. A Toolkit for Urban Innovators. London: Earthscan.

Lopera Rueda, J. (2020). Prácticas, saberes y apropiación del medio audiovisual en los colectivos de comunicación comunitaria de la ciudad de Medellín. LUCIÉRNAGA, 11(22), 70-83. doi:https://doi. org/10.33571/revistaluciernaga.v11n22a2

Malik, S., Chapain, C., \& Comunian, R. (2017). Rethinking cultural diversity in the UK film sector: Practices in community filmmaking. Sage Journals, 24(3), 308-329. doi:https://doi.org/10.1177/1350508416689094

Programa Ibermedia. (2020). Perú empieza el 2020 estrenando su nueva Ley de Cine. Recuperado el 29 de Octubre de 2020, de https://www.programaibermedia.com/peru-empieza-el-2020-estrenando-su-nueva-leyde-cine/

RPP: Radio Programas del Perú. (01 de Septiembre de 2017). PCM aprobó proyecto para nueva Ley de Cine. Lima, Lima, Peru. Recuperado el 24 de Octubre de 2020, de https://rpp.pe/cine/peru/pcm-aproboproyecto-para-nueva-ley-de-cine-noticia-1074038.?ref=rpp

Scott, A. J. (2006). Creative Cities: Conceptual Issues and Policy Questions. Journal of Urban Affairs, 28(1), 1-17. doi:https://doi.org/10.1111/j.0735-2166.2006.00256.x

Talavera, J. (2017). Film production in Europe. Production volume, co-production and worldwide circulation. Strasbourg: European Audiovisual Observatory. Recuperado de https://rm.coe.int/filmproductionineurope2017-j-talavera-pdf/1680788952

Vidaechea, J. (2013). U+3(i). Espacios para la emergencia y la fertilización de la creatividad: el caso de los festivales audiovisuales de Barcelona. Tesis para la obtención de Máster Oficial en Gestión Cultural, Universidad de Barcelona. Recuperado de http://hdl.handle.net/2445/47907

Vidaechea, J., \& Pareja-Eastaway, M. (2017). Outlining spaces for the emergence and fertilization of creativity: the case of audiovisual festivals in Barcelona. En M. Wagner, J. Valls-Pasola, \& T. Burger-Helmchen (Edits.), The Global Manahgement of Creativity (págs. 82-96). New York, Estados Unidos: Routledge.

Worldwrite. (2020a). About us. Recuperado el 24 de Octubre de 2020, de WorldWrite Web Site: https://www. worldwrite.org.uk/about/ 
Worldwrite. (2020b). Opportunities: Volunteering and training. Recuperado el 24 de Octubre de 2020, de Worldwrite Web Site: https://www.worldwrite.org.uk/volunteering/

Worldwrite. (2020c). Our Impact. Recuperado el 30 de Octubre de 2020, de https://www.worldwrite.org.uk/ about/our-impact/

Worldwrite. (2020d). About us: Partners \& Sponsors. Recuperado el 24 de Octubre de 2020, de Worldwrite Web Site: https://www.worldwrite.org.uk/about/sponsors/

\section{Notas}

[1] Este análisis abre el debate de cuáles son los criterios más adecuados para identificar si una ciudad es creativa o no y que industria es más creativa dentro de un sector cultural, ciudad y región. En tanto la informalidad en Lima, es una externalidad negativa que pueden invisibilizar información para reconocer su creatividad, se deja el cuestionamiento entorno a si Lima es o no una ciudad creativa.

[2] Siendo la tendencia de estos cuatro años, mayor cantidad de estrenos en cine alternativos que en las salas comerciales. Este 2019 se aprecia un transvase de la producción nacional a la cartelera comercial, siendo 34 películas en cines comerciales y 26 en circuitos alternativos (Cinencuentro, 2020).

[3] La asignación presupuestal anual mínima otorgada es de 6000 UIT. Un aumento que duplicar el otorgado en el 2017 de 3000 UIT

[4] Concluimos entonces que el modelo del genio solitario esta caduco. Y, en la actualidad, el genio es alguien socialmente interconectado.

[5] Tonamos como referencia La empresa "La Fábrica" por ser parte de la muestra de la investigación del análisis de las empresas españolas creativas (Casani, F et al, 2012). Esta investigación siguió los pasos del trabajo de Conhendet, seleccionando dos casos de análisis que son la empresa La Fabrica e llustrarte.

[6] Bansky es una artística especial porque no se sabe cuál es su verdadera identidad, ni cuál es su verdadera profesión. Pero cuya filosofía del arte contemporáneo pone en debate la creación del arte y considero que es una "fuerza impulsadora" para la creación de nuevas manifestaciones artísticas.

[7] Las once sedes del proyecto de Cine Comunitario de la Red de Microcines están ubicadas en Lima Norte, Lima Sur, Cusco, La Libertad, Puno, Piura, Ayacucho, Apurímac, Ancash, Loreto e Iquitos.

[8] Nos referíamos con comunidad local a la comunidad donde se ubican cada una de los microcines del grupo Chaski.

\section{Para citar este artículo:}

Díaz, K. (2020). Análisis de las experiencias de la Red de Microcines de Chaski (Peru) y Wordbytes de Worldwrite de Londres, en el desarrollo del sector audiovisual. Revista Luciérnaga Comunicación. Vol. 12, Núm. 23. Pp 88-103. https://doi.org/10.33571/revistaluciernaga.v12n23a4

\section{OJS. http://revistas.elpoli.edu.co/index.php/luc/issue/archive}

Link. https://www.politecnicojic.edu.co/index.php/revista-luciernaga 